\title{
Une Etude Exploratoire De La Politique D'ouverture Du Capital Aux Salariés Dans Les Entreprises Au Cameroun
}

\author{
Ousmanou Alim, Assistant Lecturer, Master of Finance \\ University of Buea Cameroun
}

doi: 10.19044/esj.2016.v12n13p329 URL:http://dx.doi.org/10.19044/esj.2016.v12n13p329

\begin{abstract}
The purpose of this work is to examine the employees ownership policy in Cameroon's companies. The information used is derived from primary data collected between November 2015 and January 2016 in 31 Public Limited Companies having opened the capital to their employees. They belong to all sectors: industry, service, agriculture, transport and trade. From the perspective of the workforce of employees, these companies are mostly large (employing more than 100 employees) and medium (employing between 20 and 100 employees). Through exploratory factor analysis and the univariate analysis, the study reveals that there are two forms of share ownership by employees in these companies: the direct form and indirect form. Similarly, the level of participation of employees in the capital of these enterprises is usually between $5 \%$ and $10 \%$ and the average level of around $7.4 \%$. The privatization of public and para-public enterprises was a major factor of the opening of capital to employees in the firms of the sample. In addition, the establishment of global employee share plan in subsidiaries of foreign companies is also one of the reasons attached to this policy. As for the benefits of implementing this policy, they are among others the improvement of productivity, economic performance and social performance of these companies.
\end{abstract}

Keyswords: Employee ownership, Privatization, Productivity, Economic Performance, Social Performance

\section{Résumé}

L'objet de ce travail est d'examiner la politique d'ouverture du capital aux salariés dans les entreprises au Cameroun. L'information utilisée est issue des données primaires collectées entre Novembre 2015 et Janvier 2016 dans 31 Sociétés Anonymes ayant ouvert le capital à leurs salariés. Ces entreprises appartiennent à tous les secteurs: industrie, service,agriculture, 
transport et commerce. Du point de vue de l'effectif d'employés, ces entreprises sont en majorité de grande taille (employant plus de 100 salariés) et de moyenne taille (employant entre 20 et 100 salariés). Grâce à l'analyse factorielle exploratoire et à l'analyse univariée, l'étude révèle qu'il existe deux formes de détention d'actions par les salariés dans ces entreprises : la forme directe et la forme indirecte. De même, le niveau de participation des salariés dans le capital de ces entrepises se situe généralement entre $5 \%$ et $10 \%$ et le niveau moyen autour de 7,4 \%. La privatisation des entreprises publiques et parapubliques a été un facteur majeur de l'ouverture du capital aux salariés dans les entreprises de l'échantillon. De plus, la mise en place du plan d'actionnariat salarié global dans les filiales des entreprises étrangères est aussi l'une des raisons attachées à cette politique. Quant aux avantages de la mise en place de cette politique, ils sont entre autres, l'amélioration de la productivité, de la performance économique et de la performance sociale de ces entreprises.

Mots Clés: Actionnariat salarié, Privatisation, Productivité, Performance économique, Performance sociale

\section{Introduction}

Depuis la fin des années 1980, l'actionnariat salarié fait l'objet d'une attention croissante dans le cadre de la gouvernance des entreprises. En effet, l'évolution du système de gouvernance a fait naître une nouvelle forme de participation dans les entreprises. Les salariés peuvent alors constituer un portefeuille de valeurs mobilières et acquérir dans des conditions avantageuses, des actions de la société qui les emploie (Dondi, 1992 ; Desbrières, 2002).

C'est ainsi qu'aujourd'hui, que ce soit en Europe ou aux Etats-Unis, l'actionnariat salarié prend de plus en plus de l'ampleur au sein des entreprises. En France, selon la F.A.S (Fédération des Actionnaires Salariés), il y avait en fin 1999 plus d'un million des salariés actionnaires, soit 25000 entreprises ont ouvert leur capital à leurs salariés, pour un total de 300 milliards de francs investis. Pour le trésor britannique, la quasi-totalité des entreprises de l'indice FTSE 100, avaient mis en place près de 6000 plans d'actionnariat fin 1998, soit 1750 entreprises ont ouvert le capital aux salariés, couvrant environ 2,5 millions des salariés britanniques. S’agissant du NCEO (National Center for EmployeeOwnership), en avril 2001 aux Etats-Unis, l'on dénombrait environ 11500 plans d'actionnariat en vigueur, couvrant 8,5 million des salariés pour un total de 500 milliards de dollars.

En Afrique et plus particulièrement au Cameroun, il n'existe pas de statistiques précises ; toutefois, le « Share plan 2013 d’AXA-Assurance » a permis aux 22000 salariés de cette entreprise de devenir actionnaires, dans 
38 pays dont le Cameroun. De même pour le géant Français de laboratoire SANOFI les plans d'actionnariat salarié sont importants pour fédérer les salariés du groupe en développant un sentiment d'appartenance à cette firme. Ces plans qui offrent aux salariés l'opportunité d'investir à moyen/long terme dans les actions de l'entreprise, alignent les intérêts des actionnaires et des salariés. Le dernier plan d'actionnariat salarié a été mis en place en 2013. Dans ce cadre, près de 15000 salariés dans plus de 80 pays (y compris le Cameroun) ont souscrit à près de 1,7 million d'actions.

L'objet de cette étude tourne autour du problème d'analyse de la politique d'ouverture du capital aux salariés dans les entreprises au Cameroun. Plusieurs questionnements guident cette étude : quelles sont les caractéristiques des entreprises ayant ouvert le capital à leurs salariés au Cameroun? quel est le niveau de participation des salariés dans le capital ? quelle est la forme de détention d'actions par les salariés? quelles sont les raisons qui justifient la mise en place de l'actionnariat salarié dans ces entreprises ? quels avantages ces entreprises tirent-elles de la mise en place de cette politique? .

Cette étude se propose d'apporter les éléments de réponse à ces interrogations à travers l'analyse de la pratique de l'actionnariat salarié dans les entreprises au Cameroun. L’échantillon est constitué de 31 entreprises de tous les secteurs d'activités pratiquant une politique d'actionnariat salarié. Les outils d'analyse utilisés sont l'analyse factorielle en composante principale (ACP) et l'analyse uni variée.

Après un bref exposé de l'examen du concept de l'actionnariat salarié dans la littérature, nous décrirons la méthodologie et présenterons les résultats avant de procéder à leurs analyses.

\section{Examen du concept de l'actionnariat salarié dans la littérature}

La littérature sur l'actionnariat salarié a tout d'abord cherché à étudier ses liens avec la performance organisationnelle. Les résultats de ces études se sont avérés mitigés, bien qu'ils aboutissent globalement soit à des effets positifs, soit à des effets négligeables (Buchko, 1993 ; Gamble et al. 2002 ; Trewhitt, 2000). Cette littérature a également abouti à un consensus sur la définition de l'actionnariat salarié qui permet d'intégrer la diversité des systèmes existants. En effet, à partir des travaux de Ben-Ner et Jones (1995) ; Klein (1987) ; Long (1980) et Pierce et al. (1991), l'actionnariat salarié peut être conceptualisé en termes de degré d'actions détenues par les salariés, de valeur financière de cet actionnariat et du niveau d'influence et de participation à la prise de décision que cet actionnariat apporte aux salariés.

Toutefois, Il existe de nombreuses différences pratiques entre la simple participation aux bénéfices et l'actionnariat salarié. Nous pouvons citer par exemple le fait que la participation concerne la performance passée 
alors que l'actionnariat salarié offre des droits sur la performance future. Le niveau de risque peut également être considéré comme différent. En effet, lorsque les primes de participation aux bénéfices sont versées, leur valeur n’a pas vocation à évoluer comme c'est le cas pour les actions. Enfin, la participation financière est davantage considérée comme un élément du package de la rémunération, tant dans l'esprit des salariés que dans les négociations avec les partenaires sociaux (Pendleton et al., 2001). Rappelons enfin une différence fondamentale qui concerne la propriété du capital. L’actionnariat salarié rend les salariés copropriétaires de l'entreprise, et leur permet d'exercer, un pouvoir de décision au sein de l'entreprise.

Dans la littérature plusieurs auteurs ont tenté de trouver une définition à l'actionnariat salarié. Pour Desbrières (2002), il est l'une des formules de participation financière utilisée pour stimuler les efforts des salariés. C'est un dispositif qui permet aux salariés d'accéder au capital de l'entreprise au niveau national et international à des conditions avantageuses dans le cadre des dispositifs à l'épargne salariale. Il s'agit alors d'une opération qui permet aux salariés de constituer une porte feuille de valeur mobilière et d'acquérir dans des conditions avantageuses des actions de la société qui les emploie.

Pour Aubert (2005) l'actionnariat salarié peut être considéré comme un moyen de conciliation des intérêts quelque fois divergents de propriétaires d'une entreprise et de ses employés. Le salarié qui détient les actions de son entreprise est en effet caractérisé par son double statut: par son statut de salarié, il contribue au processus de création de valeur de son entreprise ; en tant que créancier résiduel il détient un droit de bénéficier de la valeur créée par son entreprise. L'actionnariat salarié est donc une formule qui permet de conjuguer les réalités économiques et sociales de l’organisation.

Selon Autenne (2005:7) l'actionnariat salarié est « conçu comme un dispositif visant à aligner les intérêts des salariés sur ceux des actionnaires, [...] participe d'une implication financière du personnel qui a pour effet supposé de stimuler l'effort collectif et d'obtenir ainsi un accroissement de la productivité des entreprises ». Selon le National Center for Employee Ownership (NCEO) aux Etats-Unis, l'actionnariat salarié est un plan dans lequel la majeure partie des salariés d'une entreprise possèdent des actions de leur entreprise, même s'ils ne peuvent pas exercer le droit de vote attaché aux actions et même s'ils ne peuvent les revendre avant leur départ de l'entreprise (Rosen et al., 1986).

La Fédération Française des Associations d'Actionnaires Salariés (FAS), quant à elle, définit l'actionnaire salarié comme un actionnaire qui a acquis des actions de l'entreprise qui l'emploie, lors des opérations d'offres d'actions réservées à tous les salariés de cette entreprise ; ils possèdent ses actions soit directement, soit indirectement par l'intermédiaire d'un FCPE ou 
d’une SICAV d'actionnariat salarié. Ces deux définitions de l'actionnariat salarié et de l'actionnaire salarié sont très marquées par les contextes respectifs, mais montrent tout de même que l'actionnariat salarié est un concept large qui regroupe des réalités différentes. En termes managériaux, les différences peuvent être également très importantes.

Au vue de ces définitions, nous pouvons définir l'actionnariat salarié comme l'ouverture du capital par une entreprise à ses salariés dans le but de conjuguer les intérêts économiques et sociaux aux quels elle est soumise. Il s'agit aussi de la participation des salariés dans le capital de l'entreprise qui les emploie afin d'allier les exigences économiques et sociales aux quelles cette dernière est soumise.

Des définitions ci-dessus, plusieurs aspects susceptibles d'être explorés pour une analyse exploratoire de la politique d'ouverture du capital, sont à relever. Premièrement, nous avons la forme de détention du capital. Il ressort qu'il existe deux formes de détention d'actions par les salariés : la forme directe où les salariés acquièrent directement les actions de la société qui les emploie (Desbrières, 2002 ; NCEO, 2005 ; FAS, 2006 ; Audard et Bachelard, 2009), et la forme indirecte dans laquelle les salariés acquièrent les actions de leur société par l'intermédiaire d'une société d'investissement. En France, il peut s’agir d'un Fond Commun de Placement d'Entreprise (FCPE) ou d'une Société d'Investissement à Capitaux Variable (SICAV) (FAS, 2006) et aux Etats-Unis d'un «Employee Share Ownership Plan » (ESOP) (NCEO, 2005).

Deuxièmement, nous avons le niveau de participation des salariés dans le capital. Notons qu'aucune précision sur le niveau ou seuil de participation n'est faite par référence aux définitions. Cependant, la Fédération des Actionnaire Salariés (FAS) en lançant l'Indice de l'Actionnariat Salarié (IAS) en 1999 en France, précise que les entreprises qui pratiquent un actionnariat salarié significatif doivent avoir un niveau de détention d'actions par les salariés de plus de $3 \%$ ou $25 \%$ du personnel doit détenir le capital de leur entreprise. Au Cameroun, selon le bulletin n³2 du Groupement Inter-Patronal du Cameroun (GICAM, 2006) le niveau de participation des salariés dans le capital se situe entre $3 \%$ et $5 \%$ du capital dans les entreprises privatisées. Même si en contexte camerounais, Ngongang (2013) a trouvé un niveau moyen de participation des salariés dans le capital de 2,9 \% dans les entreprises, le niveau minimum de participation devrait correspondre à 3\% par référence au GICAM.

Le troisième aspect est lié à l'existence des raisons justifiant la mise en place de la politique d'ouverture du capital aux salariés dans les entreprises. Même si de façon explicite ces raisons ne ressortent pas de ces définitions, on peut en deduire quelques unes en plus de celles généralement 
mentionnées dans la litérature spécialisée. Entre autres raisons, nous pouvons citer :

- $\quad$ Les raisons d'ordre fiscal et financier, ces raisons peuvent porter sur le contrôle du capital (mise en place de l'actionnariat salarié dans le cadre des Offres Publiques d'Achat (OPA) hostiles) (Arnould et Jaeger, 1990), l'autofinancement (mise en place de l'actionnariat salarié dans le cadre de la mobilisation financière) (Dondi, 1994), la privatisation comme c'est le cas en France dans les années 80 et au Royaume-Uni (Pendleton, 2001). ;

- $\quad$ La réalisation d'économie fiscale (Maillard, 1993) ;

- $\quad$ La politique de gestion des ressources humaines, il s’agit ici de créer un esprit d'actionnaire chez les salariés (Lanciaux, 2001 ; Dondi, 1994), d’intégrer, fédérer et créer une culture commune (Lanciaux, 2001) et de concevoir une politique de rémunération motivante et attractive (Desbrières, 2002).

Le dernier aspect concerne les avantages de la mise en place de l'actionnariat salarié par ces entreprises. Il ressort de ces définitions que l'actionnariat salarié peut favoriser l'amélioration de la qualité de production et l'accroissement de la productivité (Blasi, 1988 ; Kruse, 1992 ; Jones et Kato (1995). De même, la mise en place de cette politique peut influer sur la performance économique en améliorant le résultat et la rentabilité (Fitzroy et Kraft, 1987 ; Mehran, 1999) et en augmentant la motivation et l'implication des salariés (Long, 1980 ; Gamble et al., 2002 ;Caramelli, 2006). C’est dans ce même ordre d’idée qu’Aubert (2005) précise que l'actionnariat salarié contribue au processus de création de valeur de son entreprise. C'est une formule qui permet de conjuguer les réalités économiques et sociales de l'organisation. Ainsi, l'actionnariat salarié peut agir sur la performance sociale de l'organisation à travers la réduction de l'absentéisme (Caramelli, 2006), le développement de coopération, d’une perception d’intérêt commun et de buts partagés (Gamble et al.,2002).

Relativement à ces aspects, quelles observations peut-on faire en contexte camerounais ? Pour répondre à cette question nous adoptons une méthodologie que nous décrivons dans la section qui suit.

\section{Méthodologie de la recherche}

La méthodologie développée consiste à examiner la pratique de l'actionnariat salarié dans les entreprises au Cameroun, grâce à un échantillon de 31 entreprises disposant de la politique d'actionnariat salarié, à partir des variables opérationnalisées sur la base des travaux antérieurs. Des analyses statistiques explicative et descriptive, reposant d'une part, sur l'analyse factorielle exploratoire et d'autre part, sur l'analyse univariée ont été mises en œuvre à cette fin. 


\section{L'échantillon}

L’étude s'est appuyée principalement sur les sociétés publiques et parapubliques privatisées dans les années 90, dans la mesure où selon l’ordonnance n90/004 du 22 juin 1990 et le décret n90/1257 du 30 Août 1990 portant application de l’ordonnance ci-dessus; les salariés détiennent dans toutes les entreprises privatisées 3 à $5 \%$ du capital ${ }^{7}$. Il est apparu que ces entreprises pouvaient constituer, en conséquence, une base satisfaisante. Le choix s’est fait par la méthode empirique par convenance.

Cet échantillon de 24 entreprises a subi des restrictions et quelques catégories d'entreprises ont été ainsi retirées :

- Les sociétés n’ayant pas rétrocédé la part de $5 \%$ du capital appartenant aux salariés ;

- $\quad$ Les sociétés dont les salariés ont renoncé à la politique d’actionnariat salarié ;

- Les banques, établissement de crédits et sociétés immobilières compte tenu de leurs caractéristiques financières spécifiques.

Une fois ces restrictions prise en compte trois sociétés ont été éliminées de cet échantillon. Par la suite, en s’appuyant sur les annonces du plan global d'actionnariat salarié dans les filiales des grands groupes étrangers, comme le «Share plan 2013 » d'Axa-Assurance ou celui de SANOFI, 10 autres entreprises ont été ajoutées à l'échantillon ${ }^{8}$. Au final, l'étude s’appuie sur 31 entreprises disposant de la politique d'actionnariat salarié.

Les données collectées sont de sources primaires issues des questionnaires administrés auprès des responsables d'entreprises de l'échantillon sur une période d'investigation de trois mois : Novembre 2015 à Janvier 2016.

\section{La mesure des variables}

Il est présenté d'un côté, la mesure des variables de la forme de détention et du niveau de participation des salariés dans le capital et de l'autre côté les échelles permettant de mesurer les raisons et les avantages de mise en place de politique d'ouverture du capital aux salariés.

\section{La mesure du niveau de participation des salariés dans le capital}

Pour évaluer le niveau de participation des salariés dans le capital, on fait généralement appel à la propriété d'actions des salariés. En effet, dans la littérature sur l'actionnariat salarié, la propriété d'actions des salariés a été opérationnalisée soit comme variable binaire, soit comme variable continue.

${ }^{7}$ Bulletin du Gicam, 32, Avril 2006.

${ }^{8}$ A ce niveau nous avons suivi l'actualité de ces entreprises sur leur site web pendant la période 2011-2015. 
Les cas de variables binaires distinguent les salariés actionnaires, des non actionnaires au sein de la même entreprise (Buchho, 1992 ; Tréboucq, 2002 ; Poulain-Rehm, 2007), les salariés des entreprises avec actionnariat salarié, des salariés d’entreprises sans actionnariat salarié (Ros, 2001), ainsi que les salariés travaillant dans l'entreprise avant et après la mise en place d'un plan d'actionnariat salarié (Tucker et al., 1989). En ce qui concerne l'utilisation de variables continues, la propriété d’actions a été mesurée au niveau individuel comme le nombre d'actions possédées individuellement par les salariés (Pendleton, 2001), ou au niveau organisationnel comme le pourcentage de capital de l'entreprise détenu collectivement par les salariés (Klein, 1987 ; Long, 1980 ; Pendleton, 2001). Notre étude étant réalisée au niveau organisationnel, nous retenons donc la variable continue représentant le pourcentage de capital de l'entreprise détenu collectivement par les salariés. Trois niveaux sont définis: Moins de 5\% Entre $5 \%$ et $10 \%$ et 10 \% et plus (voir encadré de la variable 1 ci-dessous).

Variable 1
Nom : Pourcentage du capital détenu par les salariés
Définition : cette variable détermine la part du capital détenu par les salariés dans leur
entreprise
Opérationnalisation :
Quel pourcentage du capital est détenu par les salariés dans votre entreprise ?
$1-\quad$ Moins de $5 \%$;
$2-\quad$ Entre $5 \%$ et $10 \%$;
$3-\quad 10 \%$ et plus

\section{La mesure de la forme de détention d'actions par les salariés}

S’agissant de la forme de détention, la littérature montre que les actions peuvent être détenues directement ou indirectement au sein des fonds communs de placement (FAS, 2006; Audard et Bachelard, 2009). Ainsi; cette variable est capté par la réponse obtenue de la question liée à la variable 2 ci-dessous (voir encadré variable 2).

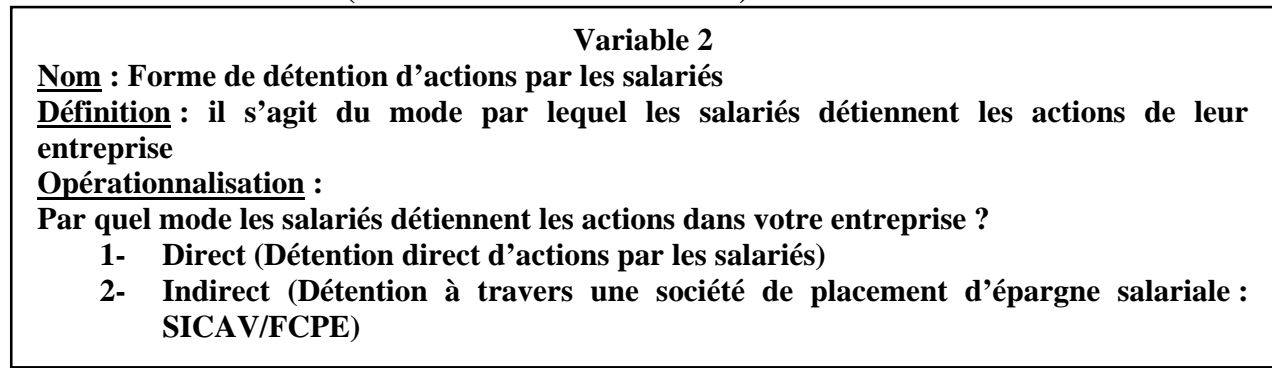

La mesure des variables portant sur les raisons de la mise en place de politique d'actionnariat salarié

En analysant la littérature sur les raisons de la mise en place d'une politique d'actionnariat salarié par une entreprise, plusieurs explications ont 
émergées à savoir les raisons d’ordre fiscal et financier (Blasi et Kruse, 1991 ; Dondi, 1992 ; Maillard, 1993 ; Pendleton, 2001) et de politique de gestion des ressources humaines (Dondi, 1994 ; Lanciaux, 2001 ; Desbrières, 2002). Ainsi, sur la base de ces explications nous avons élaboré une échelle à 8 items pour mesurer notre variable 3 .

\section{Variable 3}

Nom : Raisons de mise en place de politique d'actionnariat salarié

Définition: il s'agit des raisons qui expliquent la mise en place d'une politique d'actionnariat salarié.

Opérationnalisation : Voir l'échelle ci-dessous

Il était demandé aux répondants dans quelle mesure ils étaient d'accord avec les affirmations suivantes, selon une échelle de Likert à 5 points (1. Pas du tout d'accord, 2. Pas d'accord, 3. Avis neutre, 4. D'accord et 5. Tout à fait d'accord)

\begin{tabular}{|l|}
\hline Echelle \\
Item 1- Assurer la succession \\
Item 2- Créer un noyau dur d'actionnaires stables \\
Item 3- Réaliser une mobilisation financière \\
Item 4- Respecter les clauses de contrat de privatisation \\
Item 5- Réaliser les économies fiscales \\
Item 6- Créer chez les salariés un esprit d'actionnaire \\
Item 7- Assurer une politique d'actionnariat salarié dans votre filiale (plans globaux) \\
Item 8- Concevoir une politique de rémunération motivante et attractive \\
\hline
\end{tabular}

La mesure des variables portant sur les avantages de mise en place de cette politique par les entreprises

La littérature sur le changement de comportement et d'attitude des salariés suite à la mise en place d'une politique d'actionnariat salarié permet de déceler les avantages liés à la mise en place d'une politique d'actionnariat salarié dans les entreprises (Long, 1978a,b, ; Jones et kato, 1995 ; Caramelli, 2006). C'est sur la base de cette littérature que nous avons pu mesurer notre variable 4 à travers un ensemble de 7 items.

\section{Variable 4}

Nom : Avantages liés à la mise en place d'une politique d'actionnariat salarié

Définition: Cette variable détermine les avantages liés à la mise en place d'une politique d'actionnariat salarié.

Opérationnalisation : voir l'échelle ci-dessous.

Il était demandé aux répondants dans quelle mesure ils étaient d'accord avec les affirmations suivantes, selon une échelle de Likert à 5 points (1. Pas du tout d'accord, 2. Pas d'accord, 3. Avis neutre, 4. D'accord et 5. Tout à fait d'accord) 


\section{Echelle}

Item 1- Le contrôle des efforts des salariés actionnaires et des autres salariés Item 2- Le développement de la coopération, création d'une perception d’intérêt commun et de buts partagés

Item 3- L'accroissement de la motivation et de l'implication des salariés Item 4- La réduction de l'absentéisme et une augmentation de retour sur investissement Item 5- L'amélioration de la qualité de production Item 6- L’accroissement de la productivité Item 7- L'accroissement des résultats et de la rentabilité

\section{Les outils d'analyse statistique}

Dans un premier temps, l'analyse factorielle exploratoire sera réalisée afin de procéder à l'évaluation psychométrique des items. Il s'agit de vérifier si les items se regroupent bien de la manière prévue et de calculer aussi l'alpha de cronbach qui évalue la fiabilité de chaque échelle (Hinkin, 1995). Dans un second temps, l'analyse univariée permettra de caractériser la politique d'ouverture du capital dans les entreprises au Cameroun.

\section{Résultats de l'étude et Discussions}

La présentation des entreprises qui ont ouvert le capital à leurs salariés sera suivie de l'analyse des raisons et avantages de cette politique d'ouverture du capital aux salariés dans ces entreprises.

\section{Les caractéristiques des entreprises de l'échantillon}

L'échantillon de notre étude comporte 31 entreprises qui disposent de l'actionnariat salarié dont la majorité depuis plus de 10 ans (83,9\%). Elles sont toutes des Sociétés Anonymes (S.A) et appartiennent majoritairement au secteur industriel (45,2\%), puis aux secteurs des services (22,6\%), de l'agriculture (16,1\%), du transport (9.7\%) et du commerce (6,5\%). La majorité de ces entreprises ont 10 ans et plus d'âge (93,5\%) ; seulement 6,5 $\%$ de ces dernières ont entre 5 et 10 ans.

Selon le critère des effectifs employés, ces entreprises sont en majorité de grande taille, c'est à dire employant plus de 100 salariés (83,9 \% de l'échantillon), seulement 16,1 \% emploient entre 20 et 100 salariés (Moyennes entreprises). Pour finir, 77,4 \% de ces entreprises sont des firmes dont le capital est contrôlé par des entrepreneurs étrangers et 22,6 \% sont des firmes contrôlées par les entrepreneurs camerounais. Le Tableau 1 ci-dessous donne les caractéristiques des entreprises disposant l'actionnariat salarié. 
Tableau 1: Caractéristiques des entreprises disposant l'actionnariat salarié

\begin{tabular}{|c|c|c|c|c|c|}
\hline $\begin{array}{c}\text { Secteur } \\
\text { d'activité }\end{array}$ & $\begin{array}{c}\text { Fréquence } \\
(\%)\end{array}$ & $\begin{array}{c}\text { Age de } \\
\text { l'entreprise }\end{array}$ & $\begin{array}{c}\text { Fréquence } \\
(\%)\end{array}$ & $\begin{array}{c}\text { Effectif } \\
\text { du } \\
\text { personnel }\end{array}$ & $\begin{array}{c}\text { Fréquence } \\
\text { (\%) }\end{array}$ \\
\hline Industrie & 45,2 & 5 à 10 ans & 6,5 & $\begin{array}{l}20 \text { à } 100 \\
\text { salariés }\end{array}$ & 16,1 \\
\hline Service & 22,6 & $\begin{array}{l}10 \text { ans et } \\
\text { plus }\end{array}$ & 93,5 & $\begin{array}{c}100 \\
\text { salariés } \\
\text { et plus }\end{array}$ & 83,9 \\
\hline Agriculture & 16,1 & & & & \\
\hline Transport & 9,7 & & & & \\
\hline Commerce & 6,5 & & & & \\
\hline TOTAL & 100 & & 100 & & 100 \\
\hline
\end{tabular}

\section{Niveau de participation des salariés au capital}

Pour ce qui est du niveau de participation des salariés dans le capital, les résultats montrent que, dans la majorité des entreprises de l’échantillon le niveau de participation des salariés dans le capital se situent entre 5 et $10 \%$ (soit 61,3 \%). Toutefois dans 22,6 \% de ces entreprises ce niveau se situe en dessous de $5 \%$ et dans 16,1 \% il se situe au-dessus de $10 \%$. De même le niveau moyen de cette participation se situe à 7,4 \% du capital. Ce résultat est élevé contrairement à celui de Ngongang (2013) qui avait trouvé un niveau moyen de 2,9 \% .Ainsi, ce résultat peut s’expliquer par le fait que, la plus part des entreprises privatisées ont rétrocédé la part de $5 \%$ des salariés prévu par l’ordonnance n90/004 du 22 Juin 1990 et aussi par la mise en place des plans d'actionnariat salarié Global comme le «Share Plan 2013 d’AXA-Assurance et de SANOFI». Le tableau 2 ci-dessous présente le niveau de participation des salariés dans le capital.

Tableau 2 : Niveau de participation des salariés dans le capital de leur entreprise

\begin{tabular}{cc}
\hline Modalités & Fréquences (\%) \\
\hline Moins de $5 \%$ & 22,6 \\
Entre $5 \%$ et $10 \%$ & 61,3 \\
$10 \%$ et plus & 16,1 \\
\hline TOTAL & 100 \\
\hline
\end{tabular}

\section{La forme de détention d'actions dans les entreprises étudiées}

S'agissant de la forme, l'actionnariat salarié prend deux diverses formes dans les entreprises au Cameroun. A cet effet, les salariés détiennent des actions de leur entreprise par deux manières :

- $\quad$ Soit directement dans $74,2 \%$ des entreprises: le versement du salarié sert à souscrire directement des actions offertes par l'entreprise. Le salarié possède ses actions en détention directe au sein d'un compte titre ouvert au nominatif; elles sont bloquées pendant un certain nombre d'années. Il vote en assemblée générale, perçoit les dividendes et doit les mentionner dans sa déclaration de revenus. 
- Soit indirectement par l'intermédiaire d'une Sociétés d'Investissement à Capitaux variables d'actionnariat salarié (SICAV) dans 25,8 \% des entreprises. Le versement du salarié sert à souscrire des actions d'une Société d'Investissement à Capitaux Variables (SICAV). C'est cette SICAV qui possède et gère collectivement les actions, et le salarié les possède donc en détention indirecte. Les dividendes sont automatiquement réinvestis dans la SICAV.

A l'observation de ces résultats nous constatons que nous retrouvons les deux formes de détentions d'actions observées en France et aux EtatsUnis à savoir directement et indirectement à travers les SICAV. Par ailleurs, nous constatons que c'est la forme directe qui est plus rependue dans les entreprises de l'échantillon (soit 74,2 \% des entreprises). Ce résultat peut s'interpréter par le fait que la majorité des entreprises de l'échantillon sont des entreprises privatisées et dans ces entreprises nous retrouvons la forme directe de détention car ces dernières obéissent à la norme prévue par l'ordonnance n90/004 du 22 Juin 1990.

Le tableau 3 ci-après donne les statistiques relatives de la forme de détention d'actions par les salariés dans les entreprises de l'échantillon.

Tableau 3 : Statistiques relatives de formes de détention d'actions par les salariés

\begin{tabular}{cc}
\hline Eléments & Fréquences $(\%)$ \\
\hline Direct & 74,2 \\
Indirect (SICAV) & 25,8 \\
\hline TOTAL & 100 \\
\hline
\end{tabular}

\section{Les raisons justifiant la mise en place de la politique d'actionnariat salarié}

Le test de fiabilité de l'échelle réalisé sur l'ensemble des items donne un alpha de cronbach de l'ordre de 0,717, compris entre $\mathbf{0 , 7}<\boldsymbol{\alpha}<\mathbf{0 , 8}$ qui représente un bon seuil d'acceptabilité selon De Vellis (2003). Par la suite, nous avons procédé à l'évaluation psychométrique de cette échelle de mesure afin de vérifier que cet ensemble d'items se regroupe essentiellement pour ce concept. Pour cela, nous avons réalisé une Analyse en Composante Principale exploratoire.

Tout d'abord, le test d'adéquation des données est réalisé sur l'ensemble des données disponibles, c'est-à-dire sur l'ensemble des 31 observations concernant tous les 8 items. Un examen visuel de la matrice des corrélations Anti-image montre des corrélations faibles. Le test de précision de l'échantillonnage réalisé est le Kaiser-Mayer- Oklin Measure of Sampling Adéquacy. Il est de 0,854 donc acceptable. Le test de sphéricité de Bartlett indique un khi 2 de 154,798 avec 28 degrés de liberté et une valeur de pvalue < 0.001 : l'hypothèse nulle selon laquelle il n'y aurait aucune corrélation significative dans la matrice des données est donc rejetée. Les 
données analysées sont donc adaptées à la réalisation d’une analyse factorielle.

Nous avons réalisé une Factorisation en Axes Principaux sur l'ensemble des items. Quatre facteurs avaient une valeur propre supérieure à 1. L'examen du test de l'éboulis nous conduit à n'en retenir que deux. La variance expliquée était de 59,535. Une rotation oblique Promax a été réalisée car les facteurs sont présumés corrélés. Par mesure de précaution, seuls les items ayant une contribution factorielle supérieure ou égale à 0,7 ont été retenus dans la mesure où selon Kervin (1992), le seuil critique à partir duquel deux items sont corrélés est à partir de 0,7.

Facteur 1 : « Raisons d'ordre fiscal et financier »

Le premier facteur correspond aux raisons d'ordre «fiscal et financier ». Nous retrouvons 4 items que nous conservons pour des raisons spécifiques (contribution factorielle et alpha de cronbach).

Tableau 4 : Facteur 1, Raisons d'ordre fiscal et financier

Enoncé des Items

Réaliser une mobilisation financière

Respecter les clauses de contrat de privatisation

Réaliser les économies fiscales

Assurer une politique d'actionnariat salarié dans la filiale
Contribution factorielle

0,958

0,707

0,862

0,890

0,734

Le coefficient alpha est bon, le facteur est donc retenu.

Facteur 2: «Raisons liées à la politique de gestion des ressources humaines »

Le second facteur correspond aux raisons liées à la politique de gestion des ressources humaines du concept de « Raisons de mise en place de l'actionnariat salarié ». Il est constitué de 2 items.

Tableau 5: Facteur 2, Raisons liées à la politique de gestion des ressources humaines

\begin{tabular}{cc}
\hline Enoncé des Items & $\begin{array}{c}\text { Contribution } \\
\text { factorielle }\end{array}$ \\
\hline $\begin{array}{cc}\text { Créer chez le salarié un esprit d’actionnaire } \\
\text { Concevoir une politique de rémunération motivante et } \\
\text { attractive }\end{array}$ & 0,948 \\
\hline Alpha & 0,760 \\
\hline
\end{tabular}

Le coefficient alpha de 0.758 est bon, le facteur est retenu.

Ainsi, l'analyse descriptive sur l'ensemble des items retenus montre que : Pour ce qui est de Facteur 1, c'est-à-dire sur les raisons d'ordre fiscal et financier, deux raisons ont émergés. La première relative au respect de " clause de contrat de privatisation " a recueilli 21 avis favorables soit 67,7 \% de l'échantillon. A ce niveau il s’agit de 21 entreprises privatisées qui ont répondu à nos questionnaires et dans lesquelles l'alinéa 2 de l'article premier de l’ordonnance n90/004 du 22 juin 1990 est effectif. Ainsi, ce 
résultat est en conformité avec celui retrouvé au Royaume-Uni dans les années 80 par Pendleton et al.(2001).

La seconde raison quant à elle porte sur la mise en place d'un plan d’actionnariat salarié global, c'est-à-dire «Assurer une politique d'actionnariat salarié dans la filiale » dont nous avons obtenu 10 avis favorables qui correspondent à $32,3 \%$ de l'échantillon. Ce résultat correspond aux 10 filiales de multinationales qui ont bénéficié de la mise en place d'un plan d'actionnariat salarié global. Le tableau 6 ci-dessous présente les statistiques descriptives de raisons d'ordre fiscal et financier de mise en place d'actionnariat salarié.

Tableau 6 : Statistiques descriptives de raisons d'ordre fiscal et financier

\begin{tabular}{|cc}
\hline \multicolumn{1}{c}{ Enoncé des Items } & Fréquence (\%) \\
\hline Respecter les clauses de contrat de privatisation & 67,7 \\
Assurer une politique d'actionnariat salarié dans la filiale & 32,3 \\
\hline Total & $\mathbf{1 0 0}$ \\
\hline
\end{tabular}

Pour ce qui est de Facteur 2, faisant état des raisons liées à la politique de gestion des ressources humaine, il ressort un double motif dont l'objectif est de créer chez le salarié un esprit d'actionnaire et de concevoir une politique de réumération motivante et attractive chacun de ces items ont reçu 87,1 \% d'avis favorable dans les entreprises de l'échantillon. Ce résultat est en conformité avec les motifs trouvés par Dondi (1994) et Desbrières (2002) en France. L’illustration de ces motifs est relevée dans les statistiques élaborées dans le tableau 7.

Tableau 7 : Statistique relatives aux raisons liées à la politique de gestion des ressources humaines

\begin{tabular}{cc} 
Enoncé des Items & $\begin{array}{c}\text { Fréquence } \\
(\%)\end{array}$ \\
\hline Créer chez le salarié un esprit d’actionnaire & 87,1 \\
Concevoir une politique de rémunération motivante et attractive & 87,1 \\
\hline
\end{tabular}

\section{Les avantages de mise en place de politique d'actionnariat salarié}

Le test de fiabilité réalisé sur l'ensemble des items donne un alpha égal à 0,856 qui représente un très bon seuil d'acceptabilité selon De Vellis (2003). De même, afin de procéder à l'analyse factorielle, le test d'adéquation des données est réalisé sur l'ensemble des observations concernant tous les items. Une analyse visuelle de la matrice des corrélations Anti-image montre généralement des corrélations très faibles. L'indice KMO (Kaiser-Meyer- Oklin) de l'ordre de 0,789 est acceptable. Le test de sphéricité de Bartlett montre un Khi deux égal à 44,741 avec 21 degrés de liberté et une valeur de $\mathrm{p}=0.001$ qui indique que l'hypothèse nulle selon laquelle il n'y aurait pas de corrélation significative dans la matrice des données est par conséquent rejetée . Les données analysées sont donc factorisables. 
L'analyse en Composante Factorielle révèle trois facteurs qui disposent une valeur propre supérieure à 1 . A la suite de l'examen du test de l'éboulis, ces trois facteurs semblent plus pertinent d'où leurs conservations ; la variance expliquée est de 67,04 \%. Les facteurs étant présumés corrélés une rotation oblique Promax a été réalisée ; c'est dans ce cadre que par mesure de précaution, seuls les items ayant une contribution de factorielle supérieure ou égale à 0,7 ont été retenus.

Facteur 1 : « les avantages liés à l'amélioration de la productivité »

Le premier facteur correspond aux avantages liés à l'amélioration de la productivité des entreprises dotées de la politique d'actionnariat salarié. Il est constitué de deux items dont nous avons les raisons de les retenir.

Tableau 8: Facteur 1, les avantages liés à l'amélioration de la productivité

Enoncé des Items

Amélioration de la qualité de production

Accroissement de la productivité

Alpha
Contribution factorielle

0,785

0,794

0,701

Le coefficient est acceptable, le facteur est retenu.

Facteur 2: "les avantages liés à la performance économique »

En raison avec les avantages liés à la performance économique, le second facteur comprend trois items détaillés dans le tableau ci-dessous.

Tableau 9 : Facteur 2, les avantages liés à la performance économique

\begin{tabular}{cc}
\hline Enoncé des Items & $\begin{array}{c}\text { Contribution } \\
\text { factorielle }\end{array}$ \\
\hline Contrôle des efforts des salariés actionnaires et des autres salariés & 0,853 \\
Accroissement de la motivation et de l'implication des salariés & 0,861 \\
Accroissement des résultats et de la rentabilité & 0,703 \\
\hline Alpha & 0,741 \\
\hline
\end{tabular}

Il ressort que le coefficient alpha de l'ordre de 0,741 est bon, le facteur est retenu.

Facteur $3:$ « les avantages liés à la performance sociale »

Le troisième facteur quant à lui représente les avantages liés à la performance sociale. Il dispose de deux items dont l'alpha égal à 0,7405 est bon. Nous retenons alors ce facteur et le tableau 10 suivant présente ces items avec leur contribution factorielle.

Tableau 10 : Facteur 3, les avantages liés à la performance sociale

\begin{tabular}{cc}
\hline Enoncé des Items & $\begin{array}{c}\text { Contribution } \\
\text { factorielle }\end{array}$ \\
\hline $\begin{array}{c}\text { Développement de la coopération, création d'une perception } \\
\text { d'intérêt commun et de buts partagés }\end{array}$ & 0,727 \\
$\begin{array}{c}\text { Réduction de l'absentéisme et une augmentation du retour } \\
\text { sur investissement }\end{array}$ & 0,754 \\
\hline Alpha & 0,701 \\
\hline
\end{tabular}


A l'issu de l'examen statistique réalisé sur l'ensemble de ces items, il se dégage un certain nombre d'éléments lié à chaque facteur développé.

D’abord pour le facteur 1 concernant les avantages liés à la productivité, la qualité de production s’est améliorée dans seulement 38,7 \% des entreprises suite à la mise en place d'une politique d'actionnariat salarié. Par contre, une augmentation de la productivité dans 96,8 \% de ces entreprises est notée. Ce résultat peut s'expliquer par le niveau de participation des salariés dans le capital, dont le niveau moyen se situe à 7,4 \% contrairement à Ngongang (2013) qui avait un niveau moyen de 2,9 \% et avait noté l'absence de relation entre la participation des salariés dans le capital et la productivité D’ailleurs, Dondi (1994) et Guédri et Hollandts (2008a) ont pu démontré empiriquement le lien positif et significatif entre le niveau de participation des salariés dans le capital et la productivité.

Le tableau 11 montre les statistiques relatives aux avantages liés à la productivité.

Tableau 11 : Statistiques relatives aux avantages liés à la productivité

\begin{tabular}{cc}
\hline Enoncé des Items & Fréquence (\%) \\
\hline Amélioration de la qualité de production & 38,7 \\
Accroissement de la productivité & 96,8 \\
\hline
\end{tabular}

Ensuite, pour ce qui est des avantages liés à la performance économique, dans 87,1 \% des entreprises la mise en place de politique d’actionnariat salarié a permis le contrôle des efforts des salariés actionnaires et des autres salariés. De même, l'accroissement de la motivation et de l'implication des salariés a été ressenti dans 93,5 \% de ces entreprises et l'accroissement des résultats et de la rentabilité dans 96,7 \% de l’échantillon.

Ce résultat semble être en conformité avec celui d’Aubert (2005) qui précise que l'actionnariat salarié contribue au processus de création de valeur de son entreprise. C'est une formule qui permet de conjuguer les réalités économiques et sociales de l'organisation. Le tableau 12 suivant donne les statistiques relatives à ces avantages

Tableau 12 : Statistiques relatives aux avantages liés à la performance économique

\begin{tabular}{cc}
\hline Enoncé des Items & Fréquence (\%) \\
\hline Contrôle des efforts des salariés actionnaires et des autres & 87,1 \\
salariés & \\
Accroissement de la motivation et de l'implication des salariés & 93,5 \\
Accroissement des résultats et de la rentabilité & 96,7 \\
\hline
\end{tabular}

Enfin, concernant les avantages liés à la performance sociale, nous relevons que la réduction de l'absentéisme et le retour sur investissement est présent dans 51,6 \% des entreprises ; tandis que le développement de la coopération, création d'une perception d'intérêt commun et de buts partagés dans est ressenti dans $90,3 \%$ de ces entreprises. 
Ce résultat est en conformité avec celui de Caramelli (2006), qui dans son étude a pu prouver empiriquement que l'actionnariat salarié permet la réduction de l'absentéisme. De même pour Gamble et al. (2002) l'actionnariat salarié permet le développement de la cooépration, création d'une perception d'intérêt commun et de buts partagés. Le tableau 13 cidessous donne les statistiques relatives à ces avantages.

Tableau 13 : Statistiques relatives aux avantages liés à la performance sociale

Enoncé des Items

Développement de la coopération, création d’une perception d’intérêt commun et de buts partagés

Réduction de l'absentéisme et une augmentation du retour sur investissement
Fréquence (\%)

90,3

51,6

\section{Conclusion}

L’objet de cette étude était d'analyser la pratique de l'actionnariat salarié dans les entreprises au Cameroun. Plus précisément, en nous appuyant sur l'analyse factorielle exploratoire et l'analyse univariée nous avons essayé d'examiner la forme de détention d'action par les salariés, le niveau de participation des salariés dans le capital, les raisons et les avantages de l'ouverture du capital aux salariés dans les entreprises au Cameroun.

Globalement, deux formes d'ouverture du capital aux salariés ont émergé dans les entreprises de l'échantillon. la forme directe de détention d'actions et la forme indirecte. Mais la forme de détention directe est majoritaire dans ces entreprises. S'agissant du niveau de participation des salariés dans le capita, il se situe entre $5 \%$ et $10 \%$ dans la majorité des entreprises de l'échantillon et le niveau moyen tourne autour de 7,4 \%. Pour ce qui est des raisons de l'ouverture du capital aux salariés, deux motifs se sont distingués : le respect de clause de contrat de privatisation et la mise en place d'un plan d'actionnariat salarié global. Concernant les avantages de mise en place de cette politique, ils se situent au niveau de la productivité, de la performance économique et de la performance sociale.

Il est important de souligner à la fin de cette étude, que les résultats sont à prendre avec beaucoup de prudence. Cela d'une part, à cause de la représentativité de l'échantillon liée à la méthode d'échantillonnage (empirique par convenance) et à la taille de l'échantillon (réduite) ; d'autre part, cette étude s'est faite dans un contexte d'absence de marché financier efficient et aussi, il s'agit d'une étude exploratoire. Principalement, la méthode d'échantillonnage empirique par convenance ne nous autorise pas à généraliser les résultats. Aussi, il faut mener une étude empirique portant par exemple sur l'analyse de l'effet de l'actionnariat salarié sur la création de valeur, afin de consolider les résultats obtenus dans le cadre des avantges liés 
à la productivité, la performance économique et sociale des entreprises suite à la mise en place de l'actionnariat salarié.

\section{References:}

Aubert N. (2005). Les déterminants des investissements des salariés dans les fonds communs de placement d'entreprise d'actionnariat salarié. Thèse de Doctorat en Sciences de Gestion, Université Paul Cezanne, Aix Marseille III. Audard Let Bachelard A.(2009).L’actionnariat des salariés dans les sociétés françaises: une solution d'avenir?http://www.as-france.com/publicationasf/PrixASF/Prix-ASF-2009-actionnariat -des-salariés-dans-sociétésfrançaise.pdf

Autenne A. (2005). Quelle gouvernance des plans d'actionnariat salarié ?. Annales de Droit de l’Université Catholique de Louvain, 1-19.

Arnould D. et Jaeger M. (1990). Pratique et gestion de l'actionnariat des salariés. Revue Française de Gestion, 7-22.

Ben-Ner A. et Jones D.C. (1995). Employee participation, ownership, and productivity, a theoretical frame framework. Industrial Relations, 34, 4, 532554.

Blasi J.R. (1988). Employee ownership: revolution or rip-off? Ballinger Publishing Company.

Buchko A.A.(1992). Effects of employee ownership on employee attitudes- a test of three theoretical perspectives. Work and Occupations, 19, 1, 59-78.

Bucho A.A (1993). The effects of employee ownership on employee attitudes: an integrated causal model and path analysis. Journal of Management Studies, 30, 4, 633-657.

Caramelli M. (2006). Une étude de l'actionnariat des salariés dans le contexte de l'entreprise multinationale: une approche attitudinale interculturelle. Thèse de Doctorat en Sciences de Gestion, Université de Montpellier II

Desbrières Ph. (2002). Les actionnaires - salariés. Revue Française de Gestion, 28, 141, 225-281.

Dondi J. (1992). Contribution à la connaissance de l'actionnariat des salariés. Thèse de Doctorat en Sciences de Gestion, Université Bordeaux I.

Dondi J. (1994). L’actionnariat salarié dans les entreprises françaises : l'identification de son rôle à l'aide de résultats empiriques. Personnel, 347, 56-64.

FAS (2006).Guide de l'actionnariat salarié. $6^{\text {ème }}$ Edition.

Fitzroy F. et Kraft K. (1987). Cooperation, productivity and profit sharing. Quartely Journal of Economics, 102, 1, 23-35

Gamble J.E., Culpepper R. et Blubauch M. (2002). ESOPs and employee attitudes: the importance of empowerment and final value. Personnel Review, 31, 1, 9-26. 
GICAM (2006).Environnement de l’investissement privé au Cameroun,Bulletin $n^{\circ} 32,1-46$.

Guedri Z. et Hollandts X. (2008a) "Beyond dichotomy: The culvilinear impact of employee ownership on firm performance”, Corporate Governance : An international Review, 16, 5, 460-464.

Hinkin T.R. (1995). A review of scale development practices in the study of organizations. Journal of Management, 21, 5, 967-988.

Jones J.C. et Kato T. (1995). The productivity effects of employee ownership plans and bonuses: evidence from japanese panel data. American Economic Review, 85, 391-415.

Kervin J. B. (1992).Methods for business research. New-York: Haper Collins.

Kruse D. (1992). Profit sharing and productivity: microeconomic evidence from the United States. The Economic Journal, 102, 410, 24-36.

Klein K. (1987). Employee stock ownership and employee attitudes: A test of three models. Journal of Applied Psychology, 73, 4, 319-332.

Lanciaux C. (2001). Bâtir un plan d'actionnariat international en accord avec une stratégie d'entreprise. Personnel, 420, 33-38.

Long R.J. (1978a). The Effects of Employee Ownership on Organizational Identification, Jop Attitudes and Organizational Performance: a Tentative Framework and Empirical Findings. Human Relations, 31, 1, 29-48.

Long R.J. (1978b). The relative effects of share ownership versus control on job attitudes in an employee-owned company. Human Relations, 31, 9, 753763.

Long R.J. (1980). Job attitudes and organizational performance under employee ownership. The Academy of Management Journal, 23, 4.

Maillard P. (1993). Actionnariat des salariés : les possibilités offertes par la législation française. RECMA, 48, 61-67.

Mehran H. (1999). Unleashing the Ownership Dynamic-creating Connecting through engaged ownership-a research summary. Lincolnshire, Heweit Associates.

NCEO(2005). Données disponibles sur

http://www.nceo.org/library/eo_stat.html,10/03/2016

Ngongang D. (2013). Actionnariat salarié comme levier de création de valeur ajoutée et de productivité dans les entreprises camerounaises. Revue Gestion et Organisation, 5, 1, 1-8.

Pendleton A. (2001). Employee ownership, participation and governance: A study of ESOPs in the UK. London, New York: Routledge.

Pierce J.L., Rubenfeld S.A. et Morgan S. (1991). Employee ownership: a conceptual model of process and effects. Academy of Management Review, 16, 1, 121-144. 
Poulain-Rehm T. (2007). L’actionnariat salarié en France, un facteur de création de valeur?. Banque \& Marché, 88, 25-35..

Ros A.J. (2001). Profits for all? The costs and benefits of employee ownership. New York, USA: NOVA Science.

Rosen C., Klein K.J. et Young K.M. (1986). Employee Ownership in America, the Equity Solution. Lexintogton Books.

Tréboucq S. (2002). L’actionnariat salarié dans les entreprises familiales du SBF 250: un outil de création de valeur ?. Revue Finance Contrôle Stratégie, $15,4,107-453$.

Trewhitt L. (2000). Employee buyouts and employee involvement: a case study investigation of employee attitudes. Industrial Relations Journal, 31, 5, 437-453.

Tucker J., Nock S.L. et Toscano D.J. (1989). Employee ownership and perceptions of work. Work and Occupations, 16, 1, 26-42. 